\title{
Cooperation and Interference in Wireless Mesh Networks
}

\author{
Yuanzhu Peter Chen, Yong Wang
}

March 15, 2008

\begin{abstract}
A wireless mesh network is a multi-hop wireless network consisting of a large number of cooperative wireless nodes, which can be either stationary or mobile. The primary goal in wireless mesh networking is to achieve high system throughput in presence of numerous data traffic flows. However, because of the broadcasting nature of the wireless medium, the interference between simultaneous transmissions is a key factor affecting the throughput. Using all communication links simultaneously certainly does not guarantee high throughput of the entire network. This is because the interference between wireless links incurs unnecessary collisions and consequently retransmissions. Therefore, in order to achieve a certain level of throughput in mesh networks, the activities of these links should be arranged in a cooperative way such that a balance between interference and effective communication is attained. There have been significant research efforts to alleviate interference in wireless mesh networks and, thus, to improve the throughput. In this Chapter, we discuss existing work aiming at high throughput while simultaneously incurring minimal interference in such networks. And this is an intriguing interplay among a multitude of factors. Throughout the discussion, we focus on unicasts (one-to-one communications). We first review some of the most accepted communication and interference models in the research of multi-hop wireless networking. We then discuss the three most important approaches to minimizing interference to support multiple flows simultaneously, i.e. by routing, channel assignment, and scheduling. Afterwards, we lead the readers to the not-so-conventional approach of reducing interference by mobility. We conclude the chapter by speculating on the difficulties in modeling wireless mesh networks and the interplay of different aspects of interference avoidance.
\end{abstract}

\section{Communication and Interference Models}

When signals propagate in a wireless channel, their average strengths attenuates as a power function of the distance that they travel, which is called large-scale path loss. In addition, the signal strengths can also vary significantly even by a short distance due to propagation mechanisms such as reflection, diffraction, and scattering. As a result, when a receiver detects a signal, it is essentially receiving a combination of multiple copies of the same original signal from different paths. These multiple copies are usually modulated by different amplitudes, phases, and even frequencies. For a signal to be able to reconstruct, its strength must be 
stronger than all other adversary factors in the channel, i.e. noise and interference, by a certain factor depending on the modulation scheme used by the signal. By Shannon-Hartley Theorem, the capacity of a channel (in bps) is not more than

$$
C=B \log \left(1+\frac{S}{N}\right)
$$

where $B$ is the channel bandwidth (in Hertz) and $S / N$ is the signal-to-noise ratio. When there is interference, its strength can be included as part of the noise. Therefore, interference plays an extremely important role in wireless mesh networking, and the wireless channel must be effectively shared by different transceivers.

In order to hide the details of signal propagation in wireless channels, we need an abstract model to present to the upper layers to properly coordinate channel sharing. Ideally, such a model should be realistic enough to be useful and simple enough to be manageable. The communication and/or interference aspects of the network should be taken into account in the model. Thus, various trade-offs exist. A simplicial model of the communication capabilities of mesh networks can be represented as an undirected graph of nodes and edges. Nodes correspond to the hosts, and there is an edge between two nodes if these hosts are within range of each other. In contrast, modeling interference can be much more complicated and flexible. Various models have been proposed and adopted in the literature, including the conflict graph, interference graph, interference number, and the protocol and physical models. In practice, the relation between communication and interference is much more complex. For example, the interference is not only a spatial concept directly related to distance, but also a temporal one. In this section, we focus on the spatial aspect of interference in multihop wireless networks. We start with simple graph-based models for communication and interference. Then we move to the more realistic models that consider the strength ratio of the intended signal to interference and ambient noise. These models are used to derive the capacity upper bound in multi-hop wireless networks successfully. Essentially, the various techniques of interference-aware routing, channel assignment, and link scheduling are efforts to approach such an upper bound.

\subsection{Graph-based models}

A straightforward approach to modeling a multi-hop wireless network is using graphs to represent certain geometric properties of the network. In a graph-based model, nodes are generally assumed to be in a 2-dimensional space, and their communication capabilities and interference potentials are determined by the distance between nodes. A disk connectivity graph is a geometric graph of the nodes in the 2-D plane. We use directed graph $G=(V, E)$ to denote the disk connectivity graph, where $V$ corresponds to the nodes in the network and $E$ contains a directed edge $(u, v)$ if $v$ is within the transmission range of $u$. Graph $G$ is directed because the nodes can have different transmission ranges. As a special case, if all nodes have the same transmission range, the graph is also called a unit disk graph (UDG) [12] and it is undirected.

To incorporate interference among edges, Jain et al. [21] propose to use a conflict graph derived from the disk connectivity graph. The conflict graph $C=\left(V^{\prime}, E^{\prime}\right)$ is defined on all the links of the disk connectivity graph $G$, i.e. $V^{\prime}=E$. That is, each edge of $G$ corresponds to a vertex in $C$. Let $l_{i j}$ and $l_{p q}$ be two vertices in $C$, or two links in $G$ equivalently, where $i$, $j, p$, and $q$ are end points of the links. There is an undirected edge in $C$ between $l_{i j}$ and $l_{p q}$ 
if $\operatorname{dist}(i, q) \leq R_{i}$ or $\operatorname{dist}(p, j) \leq R_{p}$, where $\operatorname{dist}(\cdot)$ is a distance function. Here, a node $u$ in the network has an interference range $R_{u}$, within which no other node can receive a packet successfully when $u$ is transmitting. In other words, the conflict graph records that links $l_{i j}$ and $l_{p q}$ cannot be scheduled at the same time because either $q$ is within $i$ 's interference range or $j$ is within $p$ 's interference range. The degree of a vertex in the conflict graph $I$ quantize how much interference a link is susceptible to. Such is a "link-centric" interference model. Alternatively, an interference graph can also be defined on the nodes themselves. That is, the interference graph $G^{\prime}=\left(V, E^{\prime}\right)$ has a vertex for each node in the network and has a directed edge $(u, v)$ if $v$ is within $u$ 's interference range. Since the interference range of a node is always assumed to be greater than its transmission range, we know that $G$ is a spanning subgraph of $G^{\prime}$, i.e. $G \subseteq G^{\prime}$. Thus, the in-degree (out-degree, resp.) indicates how much interference a node may have as a receiver (transmitter, resp.). And this is a "node-centric" interference model, be it receiver-oriented or transmitter-oriented.

In research on topology control and power control, a goal is to minimize the graph-wise interference defined by any of the above quantities by tuning the transmission power of individual nodes so that certain graph-theoretic properties are satisfied, say connectivity and min-cut $[48,50,11,34,35]$.

\subsection{Relative signal strength based models}

In real network operation, the reception of a signal is determined by the ratio of the intended signal to the sum of ambient noise and interference, so the distance or power attenuation is not the only factor in modeling communication and interference in a wireless mesh network. Indeed, there is no such thing as transmission range or interference range in a real mesh network. Consider a transmitter and a receiver which are a short distance apart. The transmission can fail under strong noise or interference even though the separation distance is small. On the other hand, a transmitter may not garble an on-going transmission when the transmission is taking place between two sufficiently close nodes even though the interfering transmitter is not far away. Therefore, a communication or interference model should consider the relative signal strengths to be more practical. Two such models are presented by Gupta and Kumar [19], called Protocol Model and Physical Model.

1. Protocol Model - Suppose all nodes in the network have the same transmission power. The transmission from node $X_{i}$ to node $X_{j}$ is successful if

$$
\left|X_{k}-X_{j}\right| \geq|(1+\Delta)| X_{i}-X_{j} \mid
$$

for every other node $X_{k}$ in the network that is transmitting simultaneously. The value of $\Delta$ can be understood as a "guard zone" factor around the receiver $X_{j}$ to prevent any other node to from interfering with the transmission.

2. Physical Model - Let $\left\{X_{k} \mid k \in \mathcal{T}\right\}$ be the set of nodes in the network that are transmitting at the same time. Let $P_{k}$ denote the transmission power of node $X_{k}$. Then the transmission from node $X_{i}$ to $X_{j}$ is successful if

$$
\frac{\frac{P_{k}}{\left|X_{i}-X_{j}\right|^{\alpha}}}{N+\sum_{\substack{k \in \mathcal{T} \\ k \neq i}} \frac{P_{k}}{\left|X_{k}-X_{j}\right|^{\alpha}}} \geq \beta .
$$


Here, the received signal strength after path loss must be stronger than the noise and interference combined by a given threshold $\beta$, i.e. the minimum signal-to-interferenceand-noise ratio. Typically, the path loss exponent is between 2 and 4 .

There are a few differences between these two models, but the essential one is that the Protocol Model carries a "max" notion in that the interference is caused by the closest interfering node; while the Physical Model carries a "sum" notion because the interference is accumulated from all interfering nodes plus the background noise. Such a difference makes the later considerably harder to analyze mathematically. Using these two models, Gupta and Kumar are able to obtain the capacity limit of wireless mesh networks as sketched next.

\subsection{Capacity of multi-hop wireless networks}

The capacity of a wireless network is a quantification of the data transportation capabilities of the network. This is collective of all the nodes in the network because of the broadcasting nature of wireless communications. Given a network of a certain physical dimension, say a $1000 \mathrm{~m} \times 1000 \mathrm{~m}$ square, the capacity of the network is how many data can be transported by how far in a unit of time. This is an analogy of "mechanical work", and has a unit of bps $\times$ meter. Gupta and Kumar [19] first explore the capacity of multi-hop wireless networks formally. There, the network dimension is normalized to a circle of unit area, i.e. radius of $1 / \sqrt{\pi}$. Assume that there are $n$ nodes in the network sharing a channel of bandwidth $W$ bps. In addition, each node is able to control its transmission power and, thus, the communication range. Gupta and Kumar show that the network capacity is no more than $O(W \sqrt{n})$ regardless of the placement of the nodes in the network, scheduling of transceivers, and assignment of transmission power. This leads to a per-node capacity of merely $O(W / \sqrt{n})$. The intuition is illustrated in Figure 1. Suppose node $S$ wants to send a flow to node $D$. It enlists a number of intermediate notes as relays. The transmitters tune their communication ranges to $d$, indicated by the small circles. Thus, all nodes within the union of the communication ranges of the nodes on the path from $S$ to $D$ must be silent during the transportation of the flow. As a result, this flow of a length $O(1)$ "cuts" through the network in order to provide a capacity of $O(W)$ bps $\times$ meters. Consequently, there can be at most $O(\sqrt{n} / d)$ such flows active in parallel. Note that $d$ cannot be arbitrarily small to have a connected network. This capacity bound of $O(W \times \sqrt{n})$ holds for both the physical and protocol models [19]. And such a limitation comes from the shared-channel and multi-hop nature of wireless mesh networks.

To increase the capacity, two approaches have been proposed in the literature.

1. Add relay-only nodes in the network [53, 30, 27]. Relay-only nodes use an out-of-band channel to provide long-haul capacities so that the shared wireless channel is used only to provide short-range, possibly multi-hop, transportation.

2. Introduce mobility among the nodes $[8,18]$. When nodes are moving, they can "mechanically" deliver messages in the network. As a result, a much smaller number of wireless transmissions are required to deliver a message.

The first approach is relatively easy to comprehend. We will discuss the second approach later in Section 5. 


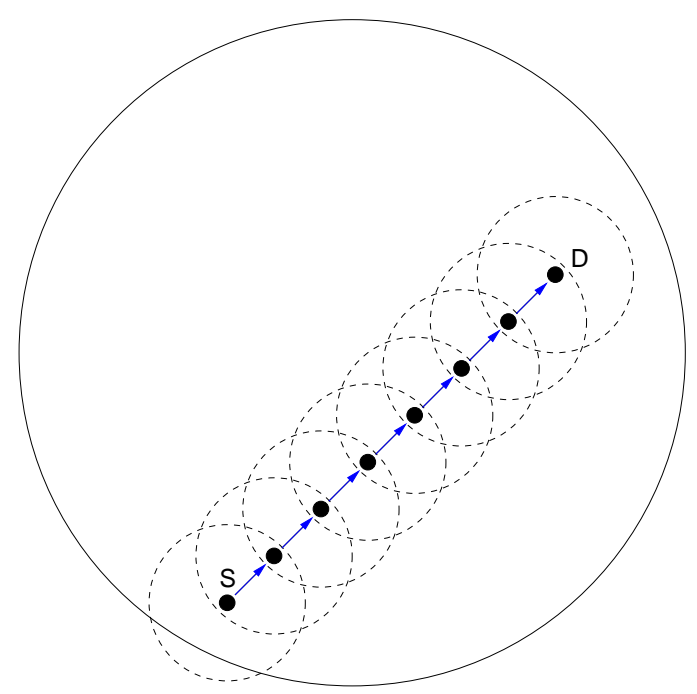

Figure 1: Capacity illustrated

\section{Interference Reduction via Routing}

Data traffic flows in mesh networks are typically multi-hop. Among different route assignments to these flows, there can be a varying degree of co-location. In order to reduce the interference among the flows, the degree of co-location should be low ideally. However, this is at the cost of consuming more network resources that could be used potentially by other traffic flows otherwise. Therefore, the selection of routes in mesh networks must consider the interference among flows, and this should be done with high adaptiveness and at low costs. In this section, we first review some background issues concerning design of routing protocols in multi-hop wireless networks. We then reflect upon the uprising awareness of minimizing interference in route calculation and packet forwarding.

\subsection{Background of routing in ad hoc and mesh networks}

Transportation of packets from the source to destination is a core enabler of any multihop network. Consequently, routing has a center role in the research of ad hoc and mesh networking. Routing has two functionality components, i.e. packet forwarding and route calculation. In packet forwarding, an intermediate node, after receiving a packet, must relay it to a neighbor based on the information contained in the packet or maintained by itself, or both. Route calculation is to prepare a node in the network, either as a packet source or forwarder, with the information that will be used to forward packets.

Apparently, design of a routing protocol can influence the network performance and software maintenance significantly. Several factors that must be considered when designing a routing protocol are listed below.

- Forwarding strategy - When a packet is received by an intermediate node, should it contain all the information needed for forwarding? This decision determines the design 
of the other factors. Such a decision differentiates a source routing protocol from the rest.

- Global structure - Is there a global structure deduced and maintained, either completely stored at different nodes or distributed separately? Under certain assumptions, global knowledge is not needed even in a distributed fashion. In this case, greedy forwarding at individual nodes can attain end-to-end packet transportation. Position-based routing forms such a category of routing protocols.

- Complete global picture - Should a single node have a complete copy of the network topology or the nodes in the network can reconstruct the complete topology collectively? Conventionally, this aspect is a distinction between link-state and distance-vector routing.

- Information acquisition - The information needed by a source node to prescribe a path for a packet or by an intermediate node to forward the packet is collected from different parts of the network. The routing protocol can require each node maintain (and help other nodes to maintain) fresh information at all times, or can wait until when such information is needed by a source. These two strategies represent proactive (tabledriven) and reactive (on-demand) routing protocols.

- Link metric - The network topology is typically represented as weighted graph of nodes. It is compiled from the link parameters reported by relevant nodes. Traditionally, hop count has been used to find a shortest path in the network. Thus, "1" is essentially reported. However, as it has been observed that more informative parameters facilitates designing a better routing protocol. These include measurements reflecting the link reliability, throughput, proximal interference, congestion level, etc.

- Cross-layer - The networking module in a node is a complex software system. For clarity in functionality definition and ease in software design and maintenance, the module is usually divided into layers, according predominantly to the ISO-OSI reference model. The cost incurred by this is however paid by the loss of efficiency of the module. Using information from different layers for decision making at a certain layer has been proved effective and efficient. Such an approach is called cross-layer design. A balance of how transparent the different layers should be is imperative at an early stage of the design.

A great deal of efforts in routing protocol design have been exerted in the research of ad hoc and mesh networking. Most of the proposals have been covered in some excellent reviews over the years $[40,10,43,1,31]$. In addition, some more surveys focus on specialized routing protocols or issues, such as position-based routing [33], multi-path routing [37], secure routing [20], and cross-layer design in routing [39]. Interested readers are referred to these articles for a comprehensive treatment.

\subsection{Increasing consciousness of interference avoidance}

When designing a routing protocol for multi-hop wireless networks, the interference among close-by nodes has been considered more and more consciously. Here, we first reflect on the changes in selecting an appropriate routing metric. These interference- and load-aware routing metrics are the basis for designing routing protocols that are able to capture the characteristics of these networks. We will review some of the most important and representative routing strategies in such endeavors subsequently. 


\subsubsection{Interference-ware metrics}

It is being realized that minimum-hop is not the best metric for route selection. Instead, reliability and medium accessibility are more descriptive measures. Earlier explorations in this direction include ABR (associativity-based routing) [49] and SSR (signal-stability based routing) [15]. In these proposals, a node broadcasts a periodic beacon as a probe. Each node evaluates the connectivity to a neighbor by counting the number of successful receptions of these beacons in recent history. SSR enhances the evaluation by measuring the signal strength experienced during reception of the probes. This idea is further refined by ETX (expected transmission count) [13]. In ETX, the bidirectional reliability between a pair of nodes is defined as

$$
\operatorname{ETX}=\frac{1}{p_{f} \times p_{r}}
$$

where $p_{f}$ and $p_{r}$ denote the probability that a transmission is successful in the forward and reverse directions, respectively. Due to the link-layer reliability requirement, this measure reflects the expected amount of time needed to complete the two-way DATA/ACK handshake. Similar to ABR and SSA, measurements are taken from periodic broadcast of probes by the nodes. The reverse link probability $p_{r}$ is recorded directly and the forward link probability $p_{f}$ is carried by the probes.

Since broadcast and unicast can be supported differently by the link layer, using a broadcast probe to evaluate a link usability for unicasts is not necessarily accurate. For example, in the IEEE 802.11 family, broadcasts are not reliable, i.e. no ACK required, whereas a unicast DATA frame should always be acknowledged by an ACK frame. In addition, a broadcast is always made at a basic data rate of the underlying physical layer protocol, but a unicast frame can be transmitted at a higher rate. Apparently, the difference in the data rates implies a difference in the BER (bit error rate), and thus in the reception success rates. In contract, per-hop round trip time (RTT) [2] is a measure based on periodic unicast frames. In the proposal, a node sends a probe to a neighbor which, upon successful reception, immediately responds with a probe acknowledgment. The primary advantage of RTT is that the contention delay experienced by the probe and its acknowledgment is the same as that experienced by any unicast frame and acknowledgment. Since both the probe and its acknowledgment are placed on the transmission queue, RTT also measures the queuing delay on both sides. In addition, the frame loss due to bad channel conditions is also included in the measurement. ETT (expected transmission time) [14] is a "bandwidth-adjusted ETX" in that

$$
\mathrm{ETT}=\operatorname{ETX} \times \frac{S}{B}
$$

where $S$ denotes the size of the packet and $B$ denotes the bandwidth of the link. Such an extension recognizes the fact that different data rates have different effects in evaluating a link's data transportation capabilities in a mesh network.

\subsubsection{Interference-aware routing}

Different approaches can be taken when designing and operating a routing protocol that battles interference in ad hoc and mesh networks. This can be done locally at an intermediate forwarder, when prescribing routes for packets, or even offline in a centralized fashion. 


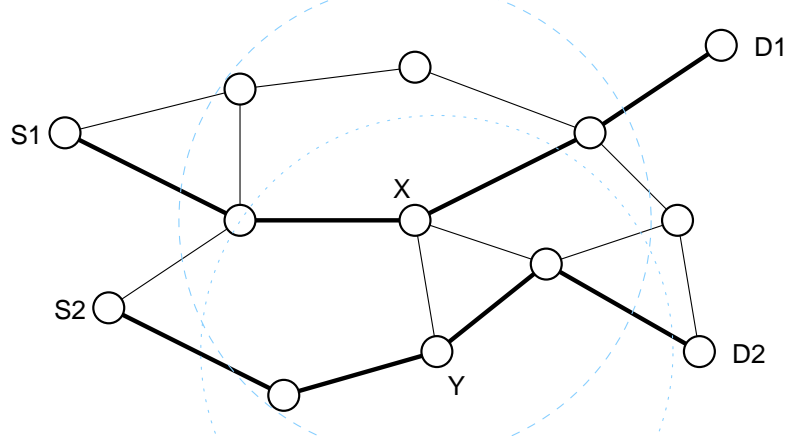

Figure 2: Inter-flow interference

After an intermediate has received a packet and is attempting to forward to a downstream neighbor, it may have difficulties in doing so because the link layer is experiencing some temporary bad channel conditions or intensive contention for that particular downstream neighbor. Rather than dropping the packet, as is conventionally done, the forwarder can choose to send it to a different neighbor as long as it is certain that the alternative neighbor can forward the packet to the ultimate destination. Such local and temporary tweak of routes in packet forwarding is called route adaptation. It is usually done jointly by the link and network layers, thus a cross-layer design. Typically, the link layer has a multicast or anycast enhancement at each node so that a forwarder can test multiple neighbors to choose one as the next forwarder of a packet. This idea has been realized in several pieces of work $[54,4,22,38]$.

Multi-path routing [37] explicitly solicits for multiple paths between a pair of source and destination. It originated from connection-oriented wired networks, such as PSTN and ATM networks, and was referred to as "alternate path routing". Its initial goal was to reduce the call blocking probability in the network core. This idea was introduced to the research of multi-hop wireless networks later in order to increase the end-to-end communication reliability and to achieve better load-balancing. Here, multiple routes are maintained and used during a single session. Depending on the independence requirement for these routes, they can be node-disjoint, link-disjoint, or non-disjoint. Apparently, the more independent these routes are from one another, the more reliable they are collectively, but the more difficult to discover and maintain. Examples of multi-path routing in such networks include SMR [29] and AOMDV $[32,22]$. Multi-path routing is not most effective in avoiding interference although is an important piece of step stone. After all, even being node-disjoint does not imply non-interfering because the interference range is usually much larger than the communication range.

For global minimization of interference among multiple flows, we can utilize centralized mathematical programming. In order to understand interference at the network layer, we can divide interference into two types: inter-flow and intra-flow.

Consider a network of nodes as in Figure 2, where the lines are the wireless links between nodes. Between two source/destination pairs $\left\{S_{1}, D_{1}\right\}$ and $\left\{S_{2}, D_{2}\right\}$, there are two flows, 


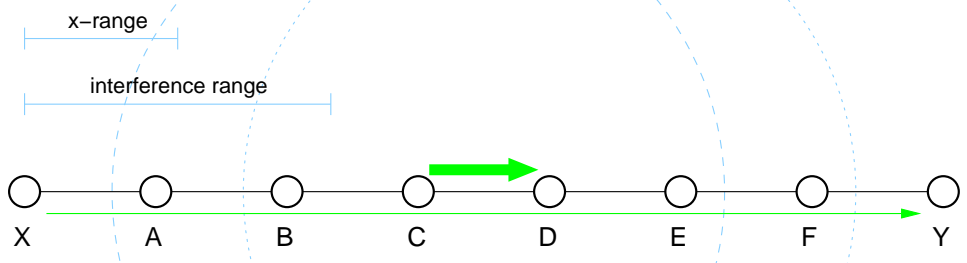

Figure 3: Intra-flow interference

indicated by solid lines. Even though the routes taken by these flows are node-disjoint, there will still be interference between them. Consider nodes $X$ and $Y$, which are within transmission range of each other. They cannot be activated at the same time because they must also be within interference range of each other, too, indicated by the dashed and dotted circles, respectively. In fact, since the interference range is considerably larger than the transmission range, even if $X$ and $Y$ were "2 hops away" in terms of transmission range, they may still be within interference range. This vital notion will be discussed more in detail shortly.

Even in a scenario of a single multi-hop flow, there is interference amongst the links comprising the route. Consider a linear topology of 8 nodes as in Figure 3 and a flow from end node $X$ to end node $Y$. In the network, the inter-nodal distance is slightly less than the transmission range. Assume that the inference range is twice of the transmission range, indicated by the circles. When node $C$ is forwarding a packet to node $D$, nodes $E$ and $F$ must be silent to avoid interfering with $D$ 's reception. Similarly, for node $C$ to receive the ACK from node $D$ successfully, nodes $A$ and $B$ must be silent, too. That is, forwarding a packet from node $C$ to $D$ is susceptible to activities on all other links en route! With these kept in mind, we will discuss what a centralized flow programming must cope with subsequently.

In a centralized route calculation, the formulation is typically based on a network flow model [3]. That is, the network is modeled with a graph with edge capacities, and a maximum combination (sum or $\lambda$-factor) of the given set of flows is pursued. The model must be enhanced to factor in the interference among and within flows as stated above. To do that, the Protocol or Physical Model (Section 1.2), or an approximate is used. In the sequel, we review some recent work provide the readers a flavor of how this is done.

Jain et al. [21] formulate the interference with a conflict graph (Section 1.1). The conflict graph derived from the connectivity graph of wireless nodes is a graph of the wireless links. In an undirected conflict graph, two vertices representing two links are adjacent if their activities interfere with each other. That is, their end points are within the interference range. In Jain et al., the optimal routing problem is formulated with a multi-commodity flow problem augmented with constraints derived from the conflict graph. They show that finding the 
optimal throughput is NP-hard even for the single source/destination case. They are able to provide upper and lower bounds on the optimum. Note that the conflict graph is indeed a simplification even for the protocol model because the interference here is determined by the absolute distance from the interferer; while in both the protocol and physical models discussed in the previous section, interference is relative to the TR (transmitter-receiver) separation distance. In this work, an attempt of using a physical model is made and shown to support similar upper and lower bounds. Again, the physical model is a simplification in that it does not consider the cumulative nature of SINR. In a later work, Kolar and Abu-Ghazaleh [26] extend to multiple flows using an interference model represented by a conflict matrix. Such a node matrix can represent an arbitrary interference relation between nodes. Again this is a protocol model not considering TR separation distance. Alicherry et al. [5] consider a joint channel assignment, scheduling and routing. The routing component therein is an interesting extension of Jain et al. [21] in itself. To model the interference, they assume that the interference range is always $q$ times greater than the communication range, where $q$ is a constant. Using the geometric properties implied, the authors show that the aggregate throughput can be approximated within a constant factor. Careful readers must have noticed that, in all these efforts, only a simplified protocol model is used to mimic the interference in the network. Using more realistic models, being it protocol or physical, makes the flow constraint specification significantly more difficult, which points an avenue for further research.

\section{Interference Reduction via Channel Assignment}

In current wireless communication technologies, a frequency band is usually divided into multiple non-overlapping sub-carriers, i.e. channels, that can operate simultaneously. Even if two sufficiently close nodes in the network are transmitting at the same time, if they use different channels, they will not interfere with each other. Such spatial frequency reuse is an important technique to avoid interference and to support multiple concurrent transmissions in multi-hop wireless networks.

There has been a transition from using a single channel to multiple channels in the research on multi-hop wireless networking. In the earlier stage, it is assumed that a single channel is used in a network and that all nodes communicate using this single channel for technological and economic reasons. With a single channel in use, interference can be avoided either by sufficient space separation or time rotation. Later in the research community, it is more accepted that a network can operate on multiple channel simultaneously. In fact, the IEEE 802.11 standard family, the most popular platform to implement wireless mesh networks, can in principle support multi-channel architectures. In particular, IEEE $802.11 \mathrm{~b} / \mathrm{g}$ provides 3 non-overlapping channels in the $2.4 \mathrm{GHz}$ band, and IEEE 802.11a provides 12 non-overlapping channels in the $5 \mathrm{GHz}$ band. Therefore, as technologies mature, using multiple channels simultaneously in a wireless mesh network is believed to be an effective and practical approach to support an increased number of concurrent transmissions, and thus to improve the throughput of the entire wireless network. In this section, we introduce two different flavors of multi-channel architectures, depending on the number of interfaces that a node has. We then go forth to categorize the channel assignment solutions into two camps according to the time granularity of channel switching. The goal of these channel assignment algorithms is unquestionably to maximize the network throughput while minimizing interference. 


\subsection{Single-NIC vs. multi-NIC}

In a multi-channel wireless mesh networks, two node configurations are possible. One is that each wireless node has one Network Interface Card (NIC), where the NIC needs to switch among the available wireless channels to fulfill different traffic demands. In the other configuration, each node has multiple NICs, each associated with one wireless channel, and can operate on these NICs at the same time. Typically, the number of NICs is assumed to be smaller than the number of available channels. We will discuss these two configurations in the sequel. In either case, each NIC at a wireless node needs to choose a channel to operate on. By carefully assigning the available channels to the NICs, interference can be reduced and, thus, the throughput of the network will be improved. When assigning channels to NICs to minimize interference, another important factor that must be considered is the connectivity of the resulting topology. For example, if an NIC is assigned a channel that is not used by any other node in range, the NIC can not communicate with the rest of the network even if there is no interference. Therefore, the channel assignment is also a trade-off between interference and connectivity.

Single-NIC multi-channel wireless mesh networks are studied in [47, 46, 7]. The main motivation for this model is that most inexpensive wireless devices in practice are equipped with a single NIC. However, the IEEE 802.11 standards do support multiple channels to enhance the network throughput. Therefore, in order to have the current widely deployed wireless hardware to take advantage of the multiple channels available, various algorithms have been proposed for single-NIC multi-channel wireless mesh networks. The approach of So and Vaidya [47] is a network layer approach, which works directly on top of the IEEE 802.11 MAC layer protocol. So and Vaidya present two heuristics to assign channels: one is to assign channels to nodes, and the other is to assign channels to traffic flows. The former separates route establishment and channel assignment, and makes the solution to the two components less complicated. However, it may cause the so called deafness problem, where the sender and receiver are not operating on the same channel. The latter considers route establishment and channel assignment together and, after a route is established, assigns all nodes on the same route a common channel. This makes the algorithm more involved but it can eliminate the deafness problem. Simulation results showed that employing multiple channels can greatly improve the network throughput compared to the single-channel architecture. The approaches taken by $[46,7]$ are MAC layer solutions, which dynamically assign channels to the NICs in an on-demand fashion. We will explore the details in the next section when we discuss the dynamic/static channel assignment.

With the development of wireless communication technologies, more and more multi-NIC wireless devices are deployed. Compared to single-NIC systems, integrating multiple NICs at each network node fits the multi-channel architecture more naturally, and can be used to further improve the network throughput. For a multi-NIC multi-channel wireless network, multiple NICs at each node are tuned to different channels and can perform communications simultaneously. In this case, a node need not switch among channels to serve one at a time. This is particularly powerful for mesh networking because relaying nodes can transport traffic continually, mitigating the intra-flow interference problem (Section 2). Raniwala et al. [42] propose a centralized algorithm for channel assignment and routing in multi-NIC multi-channel wireless networks. They use the graph-based communication and interference models (Section 1.1), where two nodes can communicate if they are within the transmission range of each other and they interfere with each other's transmission if they are within the interference range of each other, provided that the two nodes are operating on a common channel. Since channel assignment and routing are closely related to each other, and the 
strategy of one may affect the other greatly, these two problems are investigated together in Raniwala et al.. Their channel assignment consists of two phases: first, to determine through which interface a node communicates with each of its neighbors and, second, to assign a channel to each interface of a node. The second component of channel assignment interacts with the routing algorithm to refine the solution of each other in an iterated way until no improvement can be made. Their simulation results show that deploying multiple NICs at each network node can increase the network throughput significantly compared to the conventional single-channel network architecture. In a configuration of 2 NICs per node, the network throughput is increased by a factor of up to 8. In a later work, Raniwala and Chiueh [41] extend the centralized algorithm to a distributed one, which uses only local information from the $\lceil q+1\rceil$-hop neighborhood, where $q$ is the ratio between the interference and transmission ranges. Compared to the single-channel network architecture, the simulation shows that the localized algorithm can effectively coordinate a network of nodes each equipping with 2 NICs and can improve the network throughput by a factor of 6 to 7 .

Most channel assignment algorithms focus on minimizing the interference of some sort in order to improve the throughput of the network. Nevertheless, the connectivity of the resulting topology is another metric to evaluate a channel assignment algorithm. Tang et al. [48] propose a channel assignment algorithm for multi-NIC multi-channel wireless networks. Their study also uses the graph-based communication and interference models determined by the uniform transmission and interference ranges. Note that every channel assignment defines a resulting topology, where there exists an edge on channel $\gamma$ between nodes $u$ and $v$ if $u$ and $v$ are within the transmission range of each other, and if $\gamma$ is assigned to one of the NICs in node $u$ and one of the NICs in node $v$. In the resulting topology, they define the interference of a link as the number of other links which are interfere with the link, i.e. the degree of the corresponding vertex of the derived conflict graph (Section 1.1). Collectively, the interference of the resulting topology is defined as the maximum interference over all links. The proposed channel assignment algorithm in [48] strikes the balance between the interference and connectivity of the resulting topology, and generates a network structure that is interference-minimum among all $k$-connected topologies for given integer parameter $k$.

\subsection{Static vs. dynamic channel assignment}

Assigning channels can be done statically or dynamically. For static channel assignment, once a channel assignment is calculated, it will not be changed unless the topology of the network or the traffic pattern has changed significantly. In contrast, dynamic channel assignment requires that NICs switch among the available channel on demand frequently. Compared to the static approach, dynamic channel assignment captures the traffic changes more precisely and, thus, can provide better solution accordingly. However, the delay and operational costs of channel switching should also be taken into account. In addition, dynamic channel assignment algorithms need to modify the MAC layer protocols, so they cannot be deployed directly atop the off-the-shelf wireless hardware currently available. Apparently, channel assignment can also be a hybrid of static and dynamic assignment. In hybrid channel assignment, some NICs dynamically switch channels to accommodate traffic changing, while other NICs are assigned channels statically.

Channel assignment algorithms can be static in the sense that the channel assignment will be updated only when there are significant traffic or topology changes in the network. 
The static channel assignment algorithms are usually network layer, or "global", solutions, and do not need modification to the 802.11 MAC Standard. For example, the multi-NIC multi-channel work discussed earlier in this section $[42,41,48]$ are static channel assignment strategies.

Dynamic channel assignment requires that NICs be switched rapidly among channels. Such a fast switching mechanism can be challenging for node coordination. Dynamic channel assignment algorithms are usually MAC layer solutions $[7,46]$. The algorithm of So and Vaidya [46] maintains a Preferable Channel List (PCL) at each node to decide the order in selecting available channels. The PCL of a node records the use of channels inside the transmission range of the node, and is divided into different levels of preference. To accomplish a transmission request, the sender and receiver negotiate on a best channel using the PCLs of both sides, where the best is defined as the least loaded channel. That is, the algorithm tends to balance the load among different channels to reduce the bandwidth waste due to the interference and subsequent retransmissions. The algorithm proposed by Bahl et al. [7] is a slotted contention-based protocol. Each node maintains a channel schedule containing the list of channels that the node plans to switch to in subsequent time slots and the time at which it plans to make switches. When a transmission to a particular destination using a certain channel fails, back-off is adopted to reduce the probability of the further transmission conflicts.

It is also possible to combine static and dynamic channel assignment to have hybrid approaches, e.g. the hybrid channel assignment algorithms proposed in $[52,28]$. In a hybrid approach, some of the NICs in each node are constantly associated with certain pre-selected channels, and other NICs can switch among the remaining available channels. The fixed NICs are usually used to preserve the connectivity of the network topology and to exchange control information in the network. The switchable NICs are used for data transportation using channels selected dynamically. Furthermore, there are two possible settings for the NICs working on fixed channels. One is that the fixed channels are common to every node in the network [52]. Alternatively, each node has the liberty of choosing its own set of fixed channels [28]. These hybrid channel assignment algorithms are evaluated by simulations, and the results show that they do achieve a balance between flexibility and stability.

\section{Interference Reduction via Link Scheduling}

If two nodes use the same channel and are not separated sufficiently from each other, their transmission and reception must be performed at different times. Conversely, even if two nodes are rather close and they use the same channel, there will not be interference between them if well scheduled. For routing or channel assignment, as discussed previously, the network resources are usually not sufficient to accommodate all traffic demands simultaneously in an interference-free fashion. In contrast, link scheduling utilizes the much more abundant resource of time to avoid interference as long as the delay and bandwidth requirements of the users or applications are met. The idea of link scheduling is to partition time into slots, each of which is used to accommodate a subset of the given link activity requests without interference. The objective of link scheduling is usually to minimize the makespan, i.e., total number of time slots, to schedule a given set of transmissions. Here, interference can be modeled in different ways as introduced in Section 1, and this may affect the complexity of the scheduling problem significantly. Due to its similar nature to the classic CPU scheduling problems, solutions to link scheduling can resemble those to CPU scheduling. Further 
complications, however, lie in the correlation among link activation due to interference.

Currently, the research on link scheduling in wireless mesh networks has been done at the following two levels. One is to focus on the link scheduling problem itself and assume the routes and channel assignment are given. The other is to study the link scheduling problem coupled with routing and/or channel assignment. The former is relatively easier to formulate, but the latter is more effective to maximize the network throughput. The reason is that routing, channel assignment, and link scheduling are not independent subproblems for minimizing interference and maximizing throughput of the network. Instead, they are closely correlated to each other and the solution of one sub-problem does affect the decisions on the other sub-problems in a great deal. Thus, to be more practical, these sub-problems should be and often are considered together. In this section, we will discuss these link scheduling attacked at these two levels.

\subsection{Stand-alone link scheduling}

In a stand-alone link scheduling problem, a set of link activation requests are given, and the goal is to find the shortest makespan such that each request can be scheduled at least once under certain communication and interference models.

Mazumdar et al. [45] formulate the link scheduling problem as a $k$-hop matching problem in a UDG representing the network structure (Section 1.1). In graph theory, given a graph $G$, a matching is a subset of edges that do not share a common vertex. Here, this concept is generalized to " $k$-valid matching" (as termed in [45]), for any given constant $k$. In the generalization, the distance between two edges is defined as the number of hops between the two closest end-points of these edges. Then a $k$-valid matching is a set of edges such that the distance between any two of them is at least $k$. Using a simplified interference model, where two nodes can interfere with each other if and only if they are within $k$ hops in $G$, Mazumdar et al. reduce the link scheduling problem to $k$-valid match. They show that, if $k>1$, the maximum $k$-valid matching problem is NP-complete for general graphs and cannot be approximated within a constant ratio. For UDGs, it permits a Polynomial-Time Approximation Scheme (PTAS). The weighted version of the maximum $k$-valid matching problem is also studied, where the weight of each edge depends on factors such as congestion cost, supported data rate, and queue length.

More realistic interference models have been used in link scheduling. In the study of Wang et al. [51], each node has a pair of fixed but different transmission and interference ranges. The interference model used is essentially the conflict graph [21] (Section 1.1). Using this model, the links can be scheduled within a constant approximation ratio to the optimum, and the authors are able to devise a contention-based distributed algorithm to do so. Moscibroda et al. [36] study the problem under the Physical Model (Section 1.2), which better reflects the fact that a successful reception of a transmission at a receiver depends on how strong the received signal strength is compared to the interference caused by other simultaneous transmissions and the noise level. Within each time slot of the network operation, a node is either idle or transmitting at a certain transmission power level, which should be large enough to reach the intended receiver. Therefore, a valid schedule of a set of transmissions is in fact a sequence of power assignments of the nodes, where each power assignment describes the transmission power levels of all nodes in a time slot, such that all the transmissions can be accomplished successfully under the Physical Model. The scheduling complexity of a given set of link activation requests is defined as the minimum makespan over all valid schedules in 
the network. It turns out that determining the scheduling complexity is NP-complete. The authors are able to devise an algorithm which computes a schedule of length $O\left(I_{i n} \cdot \log ^{2} n\right)$, where $n$ is the number of nodes in the network and $I_{i n}$ is a static interference measure obtained from the connectivity graph defined by the maximum transmission power. The work of Brar et al. [9] is similar to [36] published at about the same time. The major difference is that in $[9]$ the link requests are weighted.

\subsection{Coupled with other approaches}

The link scheduling problem can also be formulated with routing and/or channel assignment to better improve the network throughput. Here, we provide a few such examples.

1. Joint routing and scheduling — Kodialam and Nandagopal [24] consider a joint routing and scheduling problem. They use the graph-based communication model (Section 1.1) and a simplified interference model, where the only constraint is that each node can communicate with at most one neighbor at any given time. In the communication model, each link can have a different but fixed data rate. The objective is to maximize the achievable fraction of a given set of flows specified by their sources and destinations. The authors develop an approximation algorithm with a performance ratio of $\frac{2}{3}$ compared to the upper bound obtained using a PTAS. Their experimental results show that the algorithm offers even higher throughput in practice.

2. Joint routing, channel assignment, and scheduling - A later work of Kodialam and Nandagopal [25] extends [24] by incorporating channel assignment issue as well. In their heuristics, both static and dynamic channel assignment schemes are considered and combined with routing and scheduling. According to the simulation results, both the static and dynamic approaches perform fairly well in achieving high throughput and, especially, the dynamic approach has a performance close to the optimum on average.

The joint routing, channel assignment, and scheduling problem is also studied extensively by Alicherry et al. [5]. They use a graph based communication and interference model, where the interference range is greater than the communication range by a fixed factor. It is assumed that each node has an aggregated traffic demand $l(u)$ from its associated users. The authors aim to formulate the maximum flow problem in multihop wireless networks in presence of link interference, and they are able to maximize the fraction of a given vector of node traffic loads using mathematical programming. A relaxed version of the problem is formulated as a Linear Program and optimally solved. Then in order to get the feasible solution for the original problem, some adjustments on routing and channel assignment are conducted. Finally, an interference-free link schedule is computed. The algorithm is proved to achieve solutions that are only a constant factor away from the optimal ones. Furthermore, simulation results show that the average performance is much better than the theoretical worst case bounds.

\section{$5 \quad$ Interference Reduction via Mobility}

Mobility has been an adverse factor in wireless networking for most of the time. The shadowing and multi-path fading of signal propagation can cause up to $40 \mathrm{~dB}$ of reception strength 
fluctuation in a very short period of time. In addition, mobility also creates new links and invalidate old ones when nodes change their relative positions. More recently, it has been realized that mobility can also be beneficial. The observation is that if a mobile node only transmits when it moves into the vicinity of the receiver, the interference caused by the transmission on other communication links will be decreased. The pioneering work of Grossglauser and Tse [18] shows that the capacity of a mobile ad hoc network can be increased dramatically using mobile relays. Apparently, this mechanism incurs a delay in data transportation, so a trade-off between delay and throughput should be sought.

Recall the results on the capacity of multi-hop wireless networks (Section 1.3). The major barrier to having a higher capacity in multi-hop wireless networks is that much of the traffic transmitted by a node is relayed traffic. If nodes are mobile, the situation can be fairly different. A source node can wait until it moves very close to the destination node before transmission. Indeed, the Infostation architecture [16] supports high-speed data transfer between a ground station and vehicles moving by. If nodes are not moving along roads but arbitrarily in a 2-D plane, the chance that they move very close is slim. For example, within a circle of radius $1 / \sqrt{\pi}$, the situation that two nodes are within $O(1 / \sqrt{n})$ lasts for approximately $1 / n$ fraction of time. Forcing direct transmissions from source to destination in this case can cause impractically long delay.

Grossglauser and Tse [18] then propose to use a number of relays that temporarily buffer packets until final delivery to the destination. When the number of relays is large, the chance that one copy of the packet is close to the destination comes sooner. Grossglauser and Tse show that it suffices to use a two-hop scheme for each packet. That is, the source only needs to broadcast a packet to its neighbors, which in turn transmit it to the destination when the time comes. Furthermore, this ensures that the per-node capacity in the network is constant as apposed to $O(1 / \sqrt{n})$ as in static networks.

Bansal and Liu [8] show that a bounded delay can be achieved based on a slightly different set of assumptions. Here, the unit-area network circle has $n$ static nodes and $m$ mobile nodes. The sources and destinations can be both mobile and static while the relays are mobile. In the routing algorithm, a single copy of a packet is unicast via multiple hops of relays from source to destination. At each hop, the packet is forwarded to a node that is heading to the destination approximately, until it is close enough to the destination when a last delivery is made. With sufficiently large $m$ of the order of $n$, there exists a constant $c>0$ such that the per-node throughput can be $\frac{c W \times \min \{m, n\}}{n \log ^{3} n}$, and the delay can be bounded by $\frac{4}{\sqrt{\pi}} v$ from above, where $v$ is the average velocity of the mobile nodes. More recent studies relax the requirements for the mobility models and obtain more general relation among capacity, delay and mobility [44, 17]. With these studies, using mobility to avoid interference is becoming more practical from the original, relatively theoretic framework.

\section{Conclusion and Open Issues}

Thus far, we have seen the research foci in multi-hop wireless networking from a relatively abstract point of view. We realize that, even under fairly simplified physical layer models for communication and interference, what we can achieve is not as satisfactory. The reason is that the problem is far more complex than what our models can offer. 
Building a model for upper layers of mesh networking is non-trivial, even just for individual wireless links. We all understand that the strength of a signal attenuates as it travels and that the propagation of wireless signals can be affected by reflection, diffraction, and scattering. In effect, what an antenna receives is a superposition of multiple distorted copies of the original signal mingled with additive white Gaussian noise (AWGN) and interference. Due to the stochastic nature of the above propagation mechanisms, such a superposition is random. In a relatively open area, the average received signal strength can be approximated as a power function of distance with an exponent $-4<\alpha<-2$. Unfortunately, the variance of this received power can be orders of magnitude and the operation environment are typically not open. In a real wireless mesh network, where the environments are changing even if the transceivers are not moving, these random behaviors are inevitable. How much of these are the models proposed in multi-hop wireless networking are capturing? And yet, how manageable are these models?

More difficulties come from the interaction among the activities in the network: from local interference among wireless links to global coupling of routes taken by different data traffic flows. It has been realized that load-sensitive routing in the Internet, where flows can change paths to adapt to the changing network conditions, is extremely difficult due to the convoluted correlations among the flows, queues, contention windows, etc., and to the much coarser time granularity that a countermeasure can be made compared to the rate of these changes [6]. Remember that traffic in the Internet can be much more predictable at least in an average sense. The case for multi-hop wireless networking is even worse because of the more dynamic nature of the data flows. Furthermore, interference among wireless links can only add to the complexity. We are now able to better appreciate the simplicity and beauty of TCP where billions of autonomous machines form a fairly effective feedback system, literally the largest ever created by mankind. After all, all TCP cares is the congestion window.

In order to achieve the capacity bound that a multi-hop wireless network can potentially achieve, we have discussed three techniques in the chapter, i.e. routing, channel assignment, and link scheduling. Essentially, all these are to realize an effective sharing of the communication resources through interference reduction. Note that each of these approaches to interference reduction can be applied along with other ones at the same time although they typically work at two different layers of the protocol stack. The orthogonality and dependency among these factors allow different ways to combine and separate them. Note that, to realize a higher throughput the network, there are other techniques that we are not able to cover in this chapter due to the limit of space. And they are power control and rate adaptation. In power control, a transmitter is able to tune its output power level to reach nodes at differing distances to provide sufficient SINR. Apparently, reaching farther nodes incurs stronger interference within the proximity of the transmitter. Alternatively, when the SINR is high, more sophisticated modulation schemes can be used to support higher data rates. Raising the transmission power is a way to obtain a high SINR at the receiver, but can the resultant interference be paid off by the shorter transmission time using a higher data rate? Interested readers are recommended to an intriguing discussion among these extra factors by Kim, Hou and Lim [23].

When studying wireless mesh networks, how closely do we want to resemble the real world? 


\section{References}

[1] Mehran Abolhasan, Tadeusz Wysocki, and Eryk Dutkiewicz. A review of routing protocols for mobile ad hoc networks. Ad Hoc Networks, 2(1):1-22, 2004.

[2] Atul Adya, Paramvir Bahl, Jitendra Padhye, Alec Wolman, and Lidong Zhou. A multiradio unification protocol for IEEE 802.11 wireless networks. In Proceedings of the First International Conference on Broadband Networks (BroadNets), pages 344-354, San Jose, CA, October 2004.

[3] Ravindra K. Ahuja, Thomas L. Magnanti, and James B. Orlin. Network flows : theory, algorithms, and applications. Prentice Hall, Englewood Cliffs, NJ, 1993.

[4] Jing Ai, Alhussien A. Abouzeid, and Zhenzhen Ye. Cross-layer optimal decision policies for spatial diversity forwarding in wireless ad hoc networks. In Third IEEE International Conference on Mobile Ad-hoc and Sensor Systems (MASS), Vancouver, Canada, October 2006.

[5] Mansoor Alicherry, Randeep Bhatia, and Li Erran Li. Joint channel assignment and routing for throughput optimization in multiradio wireless mesh networks. IEEE Journal on Selected Areas in Communications, 24(11):1960-1971, November 2006.

[6] Eric Anderson and Thomas E. Anderson. On the stability of adaptive routing in the presence of congestion control. In Proceedings of INFOCOM, pages 948-958, San Francisco, CA, March-April 2003.

[7] Paramvir Bahl, Ranveer Chandra, and John Dunagan. SSCH: slotted seeded channel hopping for capacity improvement in IEEE 802.11 ad-hoc wireless networks. In Proceedings of MobiCom, pages 216-230, Philadelphia, USA, September 2004.

[8] Nikhil Bansal and Zhen Liu. Capacity, delay and mobility in wireless ad-hoc networks. In Proceedings of INFOCOM, pages 1553-1563, San Francisco, CA, March-April 2003.

[9] Gurashish Brar, Douglas M. Blough, and Paolo Santi. Computationally efficient scheduling with the physical interference model for throughput improvement in wireless mesh networks. In Proceedings of MobiCom, pages 2-13, Los Angeles, CA, September 2006.

[10] Josh Broch, David A. Maltz, David B. Johnson, Yih-Chun Hu, and Jorjeta Jetcheva. A performance comparison of multi-hop wireless ad hoc network routing protocols. In Proceedings of the Fourth Annual ACM/IEEE International Conference on Mobile Computing and Networking, ACM, Dallas, TX, October 1998.

[11] Martin Burkhart, Pascal von Rickenbach, Roger Wattenhofer, and Aaron Zollinger. Does topology control reduce interference? In Proceedings MobiHoc, pages 9-19, Tokyo, Japan, May 2004.

[12] Brent N. Clark, Charles J. Colbourn, and David S. Johnson. Unit disk graphs. Discrete Mathematics, 85(1-3):165-177, 1990.

[13] Douglas S. J. De Couto, Daniel Aguayo, John Bicket, and Robert Morris. A highthroughput path metric for multi-hop wireless routing. In Proceedings of MobiCom, pages 134-146, San Diego, CA, September 2003.

[14] Richard Draves, Jitendra Padhye, and Brian Zill. Routing in multi-radio, multi-hop wireless mesh networks. In Proceedings of MobiCom, pages 114-128, Philadelphia, PA, September 2004.

[15] Rohit Dube, Cynthia D. Rais, Kuang-Yeh Wang, and Satish K. Tripathi. Signal stability based adaptive routing (SSA) for ad hoc mobile networks. IEEE Personal Communications, 4(1):36-45, February 1997.

[16] Richard H. Frenkiel, B. R. Badrinath, Joan Borras, and Roy D. Yates. The infostations challenge: balancing cost and ubiquity in delivering wireless data. IEEE Personal Communications, 7(2):66-71, April 2000.

[17] Michele Garetto, Paolo Giaccone, and Emilio Leonardi. On the capacity of ad hoc wireless networks under general node mobility. In Proceedings of INFOCOM, pages 357-365, Anchorage, Alaska, May 2007. 
[18] Matthias Grossglauser and David N. C. Tse. Mobility increases the capacity of ad-hoc wireless networks. In Proceedings of INFOCOM, pages 1360-1369, Anchorage, Alaska, April 2001.

[19] Piyush Gupta and P. R. Kumar. The capacity of wireless networks. IEEE Transactions on Information Theory, 46(2):388-404, March 2000.

[20] Yih-Chun $\mathrm{Hu}$ and Adrian Perrig. A survey of secure wireless ad hoc routing. IEEE Security \&5 Privacy, 2(3):28-39, May-June 2004.

[21] Kamal Jain, Jitendra Padhye, Venkat Padmanabhan, and Lili Qiu. Impact of interference on multi-hop wireless network performance. In Proceedings of MobiCom, pages 66-80, San Diego, CA, September 2003.

[22] Shweta Jain and Samir R. Das. Exploiting path diversity in the link layer in wireless ad hoc networks. In IEEE International Symposium on a World of Wireless Mobile and Multimedia Networks (WoWMoM), pages 22-30, Taormina, Italy, June 2005.

[23] Tae-Suk Kim, Jennifer C. Hou, and Hyuk Lim. Improving spatial reuse through tuning transmit power, carrier sense threshold, and data rate in multihop wireless networks. In Proceedings of MobiCom, pages 366-377, Los Angeles, CA, September 2006.

[24] Murali Kodialam and Thyaga Nandagopal. Characterizing achievable rates in multihop wireless networks: the joint routing and scheduling problem. In Proceedings of MobiCom, pages 42-54, San Diego, CA, September 2003.

[25] Murali Kodialam and Thyaga Nandagopal. Characterizing the capacity region in multiradio multi-channel wireless mesh networks. In Proceedings of MobiCom, pages 73-87, Cologne, Germany, August 2005.

[26] Vinay Kolar and Nael B. Abu-Ghazaleh. A multi-commodity flow approach for globally aware routing in multi-hop wireless networks. In Proceedings of the Fourth Annual IEEE International Conference on Pervasive Computing and Communications (PerCom), page 10, Pisa, Italy, March 2006.

[27] Ulas C. Kozat and Leandros Tassiulas. Throughput capacity of random ad hoc networks with infrastructure support. In Proceedings of MobiCom, pages 134-146, San Diego, CA, September 2003.

[28] Pradeep Kyasanur and Nitin H. Vaidya. Routing and link-layer protocols for multichannel multi-interface ad hoc wireless networks. ACM Mobile Computing and Communications Review (MC2R), 10(1):31-43, January 2006.

[29] Sung-Ju Lee and Mario Gerla. Split multipath routing with maximally disjoint paths in ad hoc networks. In IEEE International Conference on Communications (ICC), pages 3201-3205, St. Petersburg, Russia, June 2001.

[30] Benyuan Liu, Zhen Liu, and Don Towsley. On the capacity of hybrid wireless networks. In Proceedings of INFOCOM, pages 1543-1552, San Francisco, CA, March-April 2003.

[31] Changling Liu and Jorg Kaiser. A survey of mobile ad hoc network routing protocols. Technical Report TR-4, MINEMA, University of Magdeburg, October 2005.

[32] Mahesh K. Marina and Samir R. Das. On-demand multipath distance vector routing in ad hoc networks. In IEEE International Conference on Network Protocols (ICNP), pages 14-23, Riverside, CA, November 2001.

[33] Martin Mauve, Jorg Widmer, and Hannes Hartenstein. A survey on position-based routing in mobile ad hoc networks. IEEE Network, 15(6):30-39, November-December 2001.

[34] Kousha Moaveni-Nejad and Xiang-Yang Li. Low-interference topology control for wireless ad hoc networks. In Proceedings of the Second IEEE Communications Society Conference on Sensor and Ad Hoc Communications and Networks (SECON), Santa Clara, CA, September 2005.

[35] Thomas Moscibroda and Roger Wattenhofer. Minimizing interference in ad hoc and sensor networks. In Proceedings of DIALM-POMC, pages 24-33, Cologne, Germany, September 2005. 
[36] Thomas Moscibroda, Roger Wattenhofer, and Aaron Zollinger. Topology control meets SINR: The scheduling complexity of arbitrary topologies. In Proceedings of MobiHoc, pages 310-321, Florence, Italy, May 2006.

[37] Stephen Mueller, Rose P. Tsang, and Dipak Ghosal. Multipath routing in mobile ad hoc networks: Issues and challenges. In Edited by Maria Carla Calzarossa and Erol Gelenbe, editors, Lecture Notes in Computer Science (LNCS 2965). Springer, 2004.

[38] Vishnu Navda, Samrat Ganguly, and Samir Das. Interference-aware fast path adaptation in wireless mesh network. In Proceedings of MobiCom (poster), Los Angeles, CA, September 2006.

[39] Liang Qin and Thomas Kunz. Survey on mobile ad hoc network routing protocols and cross-layer design. Technical Report SCE-04-14, Carleton University, Ottawa, Canada, August 2004.

[40] S. Ramanathan and Martha Steenstrup. A survey of routing techniques for mobile communications networks. Mobile Networks and Applications (MONET), 1(2):89-103, 1996.

[41] Ashish Raniwala and Tzi cker Chiueh. Architecture and algorithms for an IEEE 802.11based multi-channel wireless mesh network. In Proceedings of INFOCOM, pages 22232234, Miami, FL, March 2005.

[42] Ashish Raniwala, Kartik Gopalan, and Tzi cker Chiueh. Centralized channel assignment and routing algorithms for multi-channel wireless mesh networks. ACM Mobile Computing and Communications Review (MC2R), 8(2):20-35, April 2004.

[43] Elizabeth M. Royer and Chai-Keong Toh. A review of current routing protocols for ad-hoc mobile wireless networks. IEEE Personal Communications, 6(2):46-55, April 1999.

[44] Gaurav Sharma, Ravi Mazumdar, and Ness Shroff. Delay and capacity trade-offs in mobile ad hoc networks: A global perspective. In Proceedings of INFOCOM, pages 1-12, Barcelona, Spain, April 2006.

[45] Gaurav Sharma, Ravi R. Mazumdar, and Ness B. Shroff. On the complexity of scheduling in wireless networks. In Proceedings of MobiCom, pages 227-238, Los Angeles, CA, September 2006.

[46] Jungmin So and Nitin Vaidya. Multi-channel MAC for ad hoc networks: handling multi-channel hidden terminals using a single transceiver. In Proceedings of MobiHoc, pages 222-233, Roppongi, Japan, May 2004.

[47] Jungmin So and Nitin Vaidya. A routing protocol for utilizing multiple channels in multi-hop wireless networks with a single transceiver. Technical report, UIUC Technical Report, October 2004.

[48] Jian Tang, Guoliang Xue, and Weiyi Zhang. Interference-aware topology control and QoS routing in multi-channel wireless mesh networks. In Proceedings of MobiHoc, pages 68-77, Urbana-Champaign, IL, May 2005.

[49] Chai-Keong Toh. A novel distributed routing protocol to support ad-hoc mobile computing. In Proceedings of 15th IEEE Annual International Phoenix Conference on Computers and Communications, pages 480-486, 1996.

[50] Pascal von Rickenbach, Stefan Schmid, Roger Wattenhofer, and Aaron Zollinger. A robust interference model for wireless ad hoc networks. In Proceedings IEEE WMAN, volume 13, page 239.1, Denver, CO, April 2005.

[51] Weizhao Wang, Yu Wang, Xiang-Yang Li, Wen-Zhan Song, and Ophir Frieder. Efficient interference-aware TDMA link scheduling for static wireless networks. In Proceedings of MobiCom, pages 262-273, Los Angeles, CA, September 2006.

[52] Shih-Lin Wu, Chih-Yu Lin, Yu-Chee Tseng, and Jang-Ping Sheu. A new multi-channel MAC protocol with on-demand channel assignment for multi-hop mobile ad hoc networks. In Proceedings of International Symposium on Parallel Architectures, Algorithms, and Networks (I-SPAN), pages 232-237, Dallas/Richardson, TX, December 2000. 
[53] Alexander Zemlianov and Gustavo de Veciana. Capacity of ad hoc wireless networks with infrastructure support. IEEE Journal on Selected Areas in Communications, 23(3):657-667, March 2005.

[54] Jian Zhang, Yuanzhu Peter Chen, and Ivan Marsic. MAC scheduling using channel state diversity for high-throughput IEEE 802.11 mesh networks. IEEE Communications Magazine, 46(11), November 2007. 